\title{
Development of Twisting Method of Sheet Metal using Taper Roll
}

\author{
Avanish Kumar*, Takahiro Makiyama ${ }^{\dagger}$, Shohei Kajikawa* and Takashi Kuboki* \\ The University of Electro-Communications (UEC) \\ Department of Mechanical and Intelligent Systems Engineering \\ 1-5-1 Chofu Gaoka, Chofu-shi, Tokyo, Japan \\ e-mail: avanish@mt.mce.uec.ac.jp, s.kajikawa@uec.ac.jp,kuboki@mce.uec.ac.jp \\ web page: http://www.uec.ac.jp/eng/ \\ $\dagger$ Institute of Technologists \\ Department of Mechanical and Production Engineering \\ 333 Maeya, Gyoda-shi, Saitama, Japan \\ e-mail: makiyama@iot.ac.jp \\ web page: http://www.iot.ac.jp/english/
}

\begin{abstract}
Twisted shape of metal, like a propeller or screw, are usually manufactured by machining. However, machining processes generate many metal chips, resulting in yield loss. One of the authors once proposed a new twisting method by forging [1]. However, the forging process formed a stepped surfaces and its productivity was low. This paper proposed a new processing method called, "twist rolling", which uses a pair of tapered rolls to obtain a continuous twisted shape. The effect of taper and skew angles on twist angle was investigated.

The principle of the twist rolling is described as follows: the taper rolls have a parallel section and a tapered section. A pair of the taper rolls are diagonally placed across the sheet metal. The finite element analyses (FEA) and experiments were conducted for clarifying the effect of taper and skew angles. A commercial code, ELFEN, was used for the FEA, and a prototype machine was built for the verification using aluminium sheet metals.

The FEA and experimental results were qualitatively in good agreement. The twist angle increased with the increase of the taper and skew angles. The sheet metal angle increased with the decrease of the sheet thickness. This study successfully verified the feasibility of the proposed method, "twist rolling".
\end{abstract}

\section{REFERENCES}

[1] T. Makiyama, T. Yagami, T. Teramae, G. Hirt, "New Incremental forging of Twisted Shape", COMPLAS XIII, Barcelona, p. IS-2 5-th paper. (2015) 